\title{
Nanophotonics
}

Nanophotonics.SPIEDigitalLibrary.org

\section{List of Reviewers}


The Journal of Nanophotonics would like to sincerely thank the following individuals who served as reviewers in 2017 . The success of our publication hinges on the voluntary contributions of time and energy put forth by these professionals.

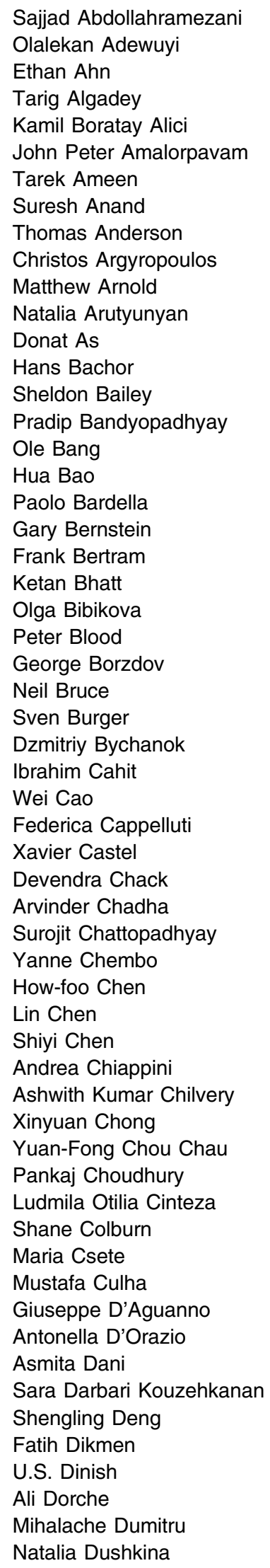

Aniruddha Dutta
Ali Eftekhar
Andrey Emelyanov
Jiyang Fan
Chao Fang
Jieran Fang
Muhammad Faryad
Chengbin Fei
Donald Fernandes
Markus Gallei
Qiaoqiang Gan
Sergey Gaponenko
Yong-kang Gong
James Grote
Jun Guan
Tuhin Guha Neogi
Hong Guo
Tarun Gupta
Yaser Hajati
Sidi Hamady
Dihan Hasan
Shlomo Hava
Ravi Hegde
Joseph Herzog
Minghui Hong
Amir Hosseini
Gao-Shan Huang
Zhifeng Huang
Dragan Indjin
Rinat Ismagilov
James Jaques
Pankaj Jha
Dengxin Ji
Jwo-Huei Jou
Taher Kagalwala
Hiroyuki Kagami
Yongqiang Kang
Tommi Kaplas
Alina Karabchevsky
Morteza Khashehchi
Nikolai Khlebtsov
Joonhee Lee
Miguel Levy
Hongin Sik Kim
Mladimir Kolesov
Oksana Kostiuchenko
Ananth Krishnan
Coleman Kronawitter
Nickolai Kukhtarev
Nardeep Kumar
Shuzhunou Li

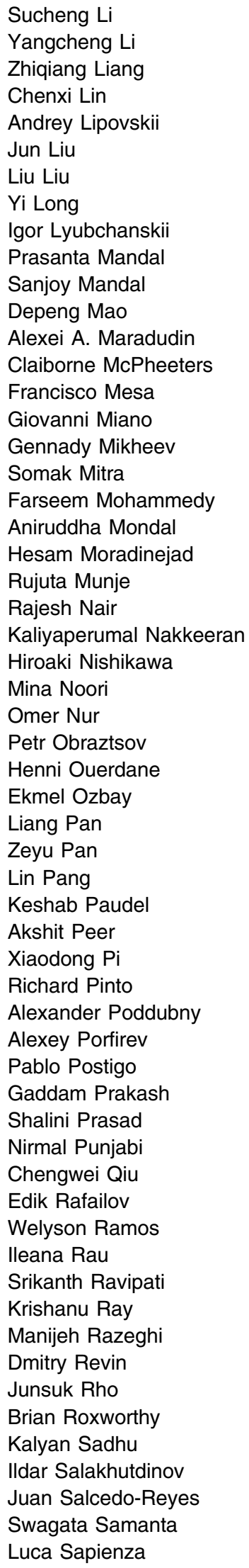


Subir Kumar Sarkar

Vasil Saroka

Malathi Sathish

Salvatore Scaglione

Mathias Schubert

Zouheir Sekkat

Stefano Selleri

Taner Sengor

Atef Shalabney

Guangcun Shan

Anuj Sharma

Mohit Sharma

Maxim Shcherbakov

Chao Shen

Yuecheng Shen

Shouyuan Shi

Alexander Shik

Makoto Shimizu

Naoto Shirahata

Poonam Shokeen

Mikhail Shuba

Kenneth Singer

Ghanshyam Singh

Rajendra Singh

Aron Sipos

Igor Smolyaninov

Hans Sohlstrom

Young-Soo Sohn

Andre Sokolnikov

Min Song

Xiaohong Song

Zhaoning Song
Evgeni Sorokin

Sachin Srivastava

Elias Stathatos

Vladimir Sugakov

Mengtao Sun

Charles Surya

Mohamed Swillam

Oleksiy Sydoruk

Mohammad Taghinejad

Osamu Takayama

Malleswara Tangi

Tino Toepper

Tomas Tolenis

Fatima Toor

Loucas Tsakalakos

Mustafa Turkmen

Darius Urbonas

Veaceslav Ursachi

Ahmad Usman

Vien Van

Carlos Villa-Angulo

Oleg Vitrik

Rami Wahsheh

Chih Ming Wang

Chunru Wang

Ken Wang

Xingjun Wang

Yanqia Wang

Yu Wang

Ziyi Wang

Jianjun Wei

Debra Weidanz
Ian White

Bodo Wilts

Anna Wojcik-Jedlinska

Chien-Jang Wu

Chunbai Wu

Jiwen Xiang

Liangxia Xie

Sajjad Yadollahzadeh

Sen Yan

Jianjun Yang

Xiaodong Yang

Yong Yang

Jie Yao

Ivan Yaskovets

Fan Ye

Fei Yi

Binxing Yu

$\mathrm{Yu} \mathrm{Yu}$

Hiroo Yugami

Alexey Yulin

Ferdows B. Zarrabi

Shuwen Zeng

Han Zhang

Peng Zhang

Yan Zhang

Jinjin Zhao

Honglou Zhen

Yuebing Zheng

Xiaolong Zhu

Zhihong Zhu 